\title{
Enhancing the Performance of Immersion Lithography: Current Challenges and Materials
}

\author{
Jae Hyun Kim \\ Manufacturing Technology Team, Infra Technology Service Center, \\ Device Solution Business, Samsung Electronics \\ San \#16 Banwol-dong, Hwasung-City, Gyeonggi-Do, Korea, 445-701
}

\begin{abstract}
Water immersion lithography is a valuable tool for sub-50-nm patterning. In efforts to improve this method, reducing immersion-specific defects in the topcoat-less process, improving scanner productivity, and enhancing the definability in the resolution-limited area are key technological challenges. Material optimization is expected to be a practical way to improve the performance of immersion lithography, thereby making the method applicable for patterning down to the sub-30-nm scale.
\end{abstract}

Keyword: immersion lithography, photoresist, top coat, dual patterning

\section{Introduction}

As device sizes have progressively decreased, the pattern density on a given area has increased considerably, while the pattern size has decreased down to about $40 \mathrm{~nm}$ for mass production. ArF $(193 \mathrm{~nm})$ immersion lithography has been widely accepted as a standard method for patterning surfaces down to the sub-50-nm scale. The method allows patterning at these dimensions without any critical problems [1]. The basic idea of immersion lithography is to fill the space between the final lens element and the photoresist with a fluid that has a much higher refractive index than air, thereby enhancing the resolution and the depth of the focus [2]. Water is widely used as a medium fluid in commercial immersion scanners, and the resolution achieved is in the sub-40-nm range.

Although immersion lithography was introduced recently, the six fundamental problems that were discussed during the initial stages of the development of this technique were solved without any significant complications [3]. Additionally, commercial tools for patterning were introduced quite rapidly for sub-50-nm node device production and development. This rapid progress in technical achievements and tool development is probably the primary reason why immersion lithography has become the main lithography technique for patterning surfaces with a resolution between sub-60 and sub- $40 \mathrm{~nm}$. Several techniques have been developed for 32-nm patterning, for example, extreme ultraviolet (EUV) lithography, high-index immersion, and immersion double patterning. Because of the manufacturability of the lutetium aluminum garnet ( $\mathrm{LuAG}$ ) lens material and the limited coverage of the node size, the high-index immersion method does not seem to be appropriate for mass manufacturing. The EUV technique also has many technical hurdles that have to be overcome, such as insufficient source power, controlling contamination in the vacuum chamber, and high mask-fabrication costs. Thus, the current 193-nm-based single- and double-immersion lithography technology will have to be applied as the main lithography tool for sub-50 to sub-20-nm patterning until a technically mature commercial EUV tool has been introduced.

\section{Defect reduction}

During the initial stages of water immersion lithography, there were many problems that seemed intractable because water is in direct 
contact with both the normal ArF photoresist and the lens element. Pattern degradation was observed and contamination of the lens occurred by leaching of photo-acid generator (PAG) molecules from the photoresist to the water medium [3]. To efficiently prevent this leaching problem, a fluoropolymer topcoat material was developed and successfully introduced into the immersion lithography process to remove any lithographic defects and solve the lens-contamination problem [4]. The main role of the topcoat material is to prevent leaching of photoresist components to the water and remove the fall-on particles that float around the inside of the track. Additionally, some people believe that the topcoat material may also reduce the density of microbridge defects, which usually increases with decreasing node size between lines.

Although the topcoat process seems to be a universal solution to all the problems observed in immersion lithography, it has two main disadvantages: 1) increased process costs due to the additional topcoat layer, and 2) patterning performance may be affected by problems stemming from the interface between the topcoat and photoresist layers. The mixed interface may lead to a worsening of the pattern profile and to a significant top loss of the photoresist film. Because of these two disadvantages of the topcoat materials, photoresist makers and process developers have tried, as much as possible, to remove the topcoat layer. Many efforts have been made to develop topcoat-less processes and immersion-specific photoresists with optimized surface characteristics that avoid the use of an additional topcoat layer $[5,6]$. However, before such immersion-specific photoresists can be applied to mass manufacturing, any possible contamination of the scanner lens has to be removed and the total number of defects from this process must be compared with the level of defects in the topcoat process.

\section{Productivity enhancement}

Although immersion lithography is the only tool for sub-50-nm patterning, an astronomical investment would be needed in order to make this technology suitable for mass manufacturing. Thus, increasing the productivity of the immersion scanner is the most important activity for device makers. During the early stages of immersion lithography, most attention was dedicated to facility stabilization and defect reduction. Having solved these initial problems, device makers are now struggling to improve the scanner productivity and to reduce the overall cost of the process.
From the viewpoint of the material contribution, there are three main factors that affect the productivity of the immersion scanner. One factor is the photosensitivity of the photoresist. Because the intensity of the scanner is limited, the scan speed for a given shot should be slowed down to reach the target intensity value for the high dose needed for the steps or layers. After the desired exposure energy has been achieved by adjusting the scanning rate for a given, the photosensitivity of the photoresist for each step should be optimized to reach the highest scanning rate for the given facility. Another factor that affects the number of wafers that can be produced in a scanner is the process temperature during the photoresist treatment. Especially, the PEB (post-exposure-bake) temperature difference between photoresists in consecutive wafer batches makes a difference in the immersion-scanner productivity. Different photoresists can have different PEB temperatures which can be optimized to show the best performance for a given process temperature. If there are two different batches that should be processed using different photoresists with different PEB temperatures, the PEB temperature of the second batch should be changed before processing. This change in the PEB temperature delays the consecutive operation of the scanner. Thus, process algorithms have to be optimized or the PEB temperature of the consecutive batches has to be unified so that they do not degrade the maximum number of wafers that can be processed in the scanner. The third factor that should be improved is the rate of rework, which has to be decreased - a modification that is increasingly difficult to implement. Normally, the rework rate increases gradually with decreasing pattern size. There could be many origins for the rework, but an appropriate photoresist can usually improve the accuracy of the process. How fast we can find the problem and how correctly we are able to solve it will be the "barometer" of how close we are to reaching optimal productivity using the immersion scanner.

\section{Beyond resolution limitation; Double Patterning Technology (DPT)}

The resolution of the current 1.35NA scanner will meet its limitation for further shrinkage at a pattern size of around $37 \mathrm{~nm}$. This upcoming resolution limit of the currently available immersion scanner for mass manufacturing has driven us to develop high-contrast photoresists with good definability. Short-diffusion-length PAG and resin systems have been designed to remove 
the chemical image blur by the diffusion of an acid to the unexposed area, consequently improving the resolution with the given optical contrast of the current scanner [7]. The negative effect observed on the line-width roughness (LWR) by the introduction of the short diffusion system was supplemented by the improvement of the PAG uniformity and the increase of the concentration of PAG molecules in the resin. The PEB temperature is also decreased to values below $90^{\circ} \mathrm{C}$ to suppress the chemical inhomogeneities resulting from both interactions between resin molecules and acid diffusion within the resin $[8,9]$. These combined activities could improve the resolution limit of current scanners and play an important role in extending the usage of single-exposure immersion patterning.

A practical extension of the current water immersion lithography process to applications down to the sub-30-nm scale will be achieved by using immersion photoresists and more complex processes with specially functionalized materials in double patterning technology (DPT) applications [10]. Currently, several viable processes are being developed to satisfy the patterning accuracy and reduce the economic costs of the process. A bar-to-space ratio of 1:3 for the pitch patterning performance and other factors, such as the LWR and the presence of defects, should be optimized in order to overcome the sub-30-nm node size patterning problems. Further materials for the second bar pattern in the middle of the first space area are still under intensive investigation.

\section{Acknowledgements}

I am grateful to my coworkers at Samsung Electronics for their everlasting passion and dedication to stabilizing the immersion scanner and improving the lithography process.

\section{References}

1. B. J. Lin, Journal of Microlithograpy, Microfabrication, and Microsystems, 3, (2004), 533.

2. S. Owa, H. Nagasaka, Journal of Microlithograpy, Microfabrication, and Microsystems, 3, (2004), 1.

3. S. Owa, H. Nagasaka, Proc. SPIE, $\mathbf{5 0 4 0}$ (2003)

4. R. D. Allen, W. Hinsberg, G. M. Wallraff, C. E. Larson, J. Meute, P. Brock, L. Sundberg, T. Chiba, T. Shimokawa, M. Slezak, J. Photopolymer Science and Technology, 18 (2005) 615

5. D. P. Sanders, L. K. Sundberg, R. Sooriyakumaran, P. J. Brock, R. A. DiPietro, H. D. Truong, D. C. Miller, M. C. Lawson, R. D. Allen, Proc. SPIE, 6519-03 (2007)

6. S. Wu, A. Tseng, B. Lin, C. C. Yu, B.-J. Lu, W.-S. Liao, D. Wang, V. Vohra, C. B. $\mathrm{Xu}, \mathrm{S}$. Caporale, G. Barclay, Proc. SPIE, 6923-05 (2008)

7. W. Hinsberg, G. M. Wallraff, C. E. Larson, B. W. Davis, V. Deline, S. Raoux, D. Miller, F. A. Houle, J. Hoffnagle, M. I. Sanchez, C. Rettner, L. K. Sundberg, D. R. Medeiros, R. R. Dammel, W. E. Conley, Proc. SPIE, 5376-21 (2004)

8. R. Sooriyakumaran, R. DiPietro, H. Truong, P. Brock, R. Allen, L. Bozano, I. Popova, W.-S. Huang, R. Chen, M. Khojasteh, P. R. Varanasi, Proc. SPIE, 6923 (2008)

9. S. Kang, B. D. Vogt, W.-L. Wu, V. M. Prabhu, D. L. VanderHart, A. Rao, E. K. Lin, K. Turnquest, Proc. SPIE, 6519-16 (2007)

10. R. D. Allen, J. Photopolymer Science and Technology, 20 (2007) 453 